\title{
Effectiveness of Structured Interventional Strategy on Knowledge, Attitude and health behavior regarding selected modifiable coronary artery disease risk factors among adolescents in selected schools
}

\author{
Associate Prof. Hepsi Rachel. A, ${ }^{1}$ Prasanna Baby ${ }^{2}$, \\ Sri Gokulam College of Nursing, (Ph.D. Scholar) Saveetha University, Chennai, India. \\ Principal, Faculty of Nursing, Sri Ramachandra University, Chennai, India.
}

\begin{abstract}
Cardiovascular diseases (CVDs) remain the biggest cause of deaths worldwide. Although CVDs typically occur in middle age or later, risk factors are determined to a great extent by behaviours learned in childhood and continued into adulthood. Adolescence provides an opportunity for teenagers to incorporate healthy lifestyle behaviours that will benefit them not only during the teenage years, but also throughout the life span. The aim of the study was to evaluate the effectiveness of structured interventional strategy on knowledge, attitude and health behavior regarding prevention of selected modifiable CAD risk factors among adolescents in selected schools. An experimental (pre-test and post-test) design was adopted for this study. 80 adolescents were randomly assigned to either experimental or control groups. A structured questionnaire, a five point likert scale, and a 4 point rating scale were used. A pre-test was administered followed by (video-assisted teaching). Three post-tests were given. The results revealed that, the overall paired ' $t$ ' test in experimental group for knowledge was 24.86, for attitude 17.97 and health behavior 27.01 which were highly significant at $P<0.001$ level. The overall unpaired ' $t$ ' test for $3^{\text {rd }}$ post-test scores on knowledge was 42.39, for attitude 16.93 and for health behavior 39.49 which were highly significant at $P<0.001$ level. There was no significant correlation between the dependent variables and there was no significant association between pre-test knowledge, attitude and health behavior and selected demographic variables except for dietary patterns.
\end{abstract}

Keywords: Adolescents, Effectiveness, Knowledge, Selected modifiable CAD risk factors, structured interventional strategy.

\section{Introduction}

Cardiovascular diseases (CVDs) remain the biggest cause of deaths worldwide of the 57 million global deaths in 2008, 36 million (63\%) were due to non-communicable diseases and 17.3 million (30\%) were due to CVDs. Nearly $80 \%$ of non-communicable diseases deaths occur in Lower Middle Income Countries (LMICs) and is the most frequent cause of death in most countries. ${ }^{(1)}$ According to the global burden of disease estimates $68 \%$ of the 751 million Years Living with Disability (YLD) worldwide is attributable to Non-communicable diseases (NCDs). ${ }^{(2)}$ While $29 \%$ of NCD deaths occurs among people below the age of 60 in LMICs, in high income countries only $13 \%$ of deaths occur below the age of $60 .{ }^{(3)}$ CVDs are responsible for 151,377 million DALYs(Disability adjusted life years) of which 62.587 million are due to coronary heart disease. ${ }^{(2)}{ }^{(4)}$ The prevalence of CAD in India has more than doubled in the past two decades. India topped the world for 1,531,534 cardiovascular disease related deaths in 2002, based on WHO reports, 2009. (5) Half of the CVD related deaths ie $52 \%$ of CVDs in India occur below the age of 50 years, and about $25 \%$ of acute myocardial infraction (MI) in India occurs under the age of 40 years. About 5-10\% of heart attacks occur in Indian men and women younger than 40 years (Mackay and Mahseh, 2004). ${ }^{(6)}$ The WHO estimates that atleast 20 million people survive CAD - related heart attacks and strokes every year around the world; by 2030, almost 23.6 million people will die from CADs mainly from heart disease and stroke. These are projected to remain the single leading cause of death. ${ }^{(7)}$

\subsection{Background:}

Atherosclerosis is the major cause of CAD. Artersiosclerosis and atherosclerosis are conditions that develop over a long period (beginning in early childhood), and progress without symptoms throughout adult life. ${ }^{(8)}$ The early changes of atherosclerosis develop in childhood and adolescence due to overall effect of a number of risk factors. ${ }^{(9)}$ Framingham research has helped define the quantitative and additive nature of these causes or, as they are now called cardiovascular risk factors. Over 300 risk factors have been associated with coronary heart disease and stroke. In developing countries with low mortality, such as China, cardiovascular risk factors also figure high on the top 10 list. Even in developing countries with high mortality, such as those in sub-saharan Africa, high blood pressure, high cholesterol, tobacco and alcohol use, as well as low vegetable and 
fruit intake, already figure among the top risk factors. Some major risks are modifiable in that they can be prevented, treated and controlled. Major modifiable risk factors are high blood pressure, high blood lipids or cholesterol, tobacco use, physical inactivity, obesity, unhealthy diets and diabetes mellitus. There are other modifiable risk factors too namely alcohol use, stress etc. The non-modifiable risk factors include, advancing age, heredity or family history, gender, ethnicity or race. ${ }^{(9)}$

\subsection{Need for the study}

Although cardiovascular diseases typically occur in middle age or later, risk factors are determined to a great extent by behaviours learned in childhood and continued into adulthood such as dietary habits and smoking. According to WHO (CVD - Atlas, 2012) worldwide, 18 million children under five years old are overweight, and $14 \%$ of 13 to 15 year old students around the world currently smoke cigarettes. Throughout the world, these risks are starting to appear earlier. Physical activity decreases markedly in adolescence, particularly in girls. In the USA physical activity decreased precipitously, especially in girls, beginning around age 10 years. ${ }^{(10)}$ The major causes of morbidity and mortality in adolescence are not diseases, but health damaging behaviours, health promotion for this age group consists mainly of teaching and guidance to avoid risk taking activities and health damaging behaviours. Adolescence provides an opportunity for teenagers to incorporate healthy lifestyle behaviours that will benefit them not only during the teenage years, but also throughout the life span. ${ }^{(11)}$ The evolution of preventive health care approach is derived from the long-standing view that the science of pediatrics is a science of health and development. Preventive care for children and youth offers greater opportunity for health care savings. The more the risk factors are identified and measured, the clearer the path we can follow to identify those adolescents at risk and engage them in reducing risks earlier. ${ }^{(12)}$ Research studies provide evidence that promoting and establishing healthy behaviors for younger people are more effective, and often easier than efforts to change unhealthy behaviours already established in adult populations. For successful prevention and control of the cardiovascular disease epidemic, health education is essential to promote health choices. Health and education are strongly linked. To maintain good health and avoid preventable disease throughout their lives, children must require knowledge about health risks and acquire skills that are needed to avoid or reduce those risks. Promoting healthy practices during adolescence, and taking steps to better protect young people from health risks is critical to the future of countries' health and social infrastructure used to the prevention of health problems in adulthood. Teaching with multimedia presentations have a certain amount of entertainment value for students. A number of studies have suggested that student satisfaction and motivation is higher that use multimedia materials (Astleither \& Wiesner, 2004). The main strategies to meet the goals and targets have included education and awareness raising, behavior change, counseling and testing, improved care and increased access to treatment. At the level of the individual, knowledge and understanding are key to behavior change. Nurses have been actively involved in the delivery of these key strategies. Media campaigns and the use of new communication technologies have been successful in increasing knowledge and changing behavior especially when complemented with education through the use of novel communication techniques. Conducting primary prevention of CVD, beginning in early childhood and sustained through adolescence has been well supported by the extensive evidence from epidemiological, clinical, and laboratory studies conducted worldwide. Hence, the researcher has chosen this method of intervention to improve their knowledge, develop a most favourable attitude and inculcate healthy behavior to cut the risk factors right at the root level.

\subsection{Objectives}

1. To assess the level of existing knowledge, attitude and health behavior among the adolescents on prevention of selected modifiable coronary artery disease risk factors in experimental and control groups.

2. To evaluate the effectiveness of structured interventional strategy on knowledge, attitude and health behavior among the adolescents on prevention of selected modifiable coronary artery disease risk factors in experimental and control groups.

3. To determine the relationship between knowledge, attitude and health behavior regarding structured interventional strategy on prevention of selected modifiable coronary artery disease risk factors among the adolescents in the experimental group.

4. To determine the association between pre-test \& post-test knowledge, attitude and health behavior among the adolescents regarding prevention of selected modifiable coronary artery disease risk factors with selected demographic variables in experimental and control group.

\section{Methodology}

A quantitative research approach was used for this study. The research design adopted was experimental research (pre-test, post-test) design. Sampling technique used was systematic random sampling. Pender's Health Promotion model was used as a conceptual framework. Data collection was done from August 
to November 2013. Data was collected using tool (1) Structured questionnaire to assess knowledge, (2) Five point likert scale to assess attitude and (3) Four point rating scale to assess the health behavior of the adolescents regarding prevention of selected modifiable CAD risk factors. Content validity and reliability was done for the three tools. After obtaining the formal permission from the school authorities, Salem (CEO, IMS \& Principals) and written consent from the adolescents, pre-test was conducted; baseline physiological parameters like BMI, hip-waist circumferences and blood pressure were measured. On the same day, a video-assisted teaching was given (to the experimental group) for 30 minutes. 3 post-tests were conducted after 1 week, 1 month and 3 months for both groups. For hypothesis testing only the $3^{\text {rd }}$ post-test score was compared with the pre-test.

\section{Result}

3.1. Section-I: Distribution of adolescents according to their demographic variables in the experimental and control group.

$52.5 \%$ of the adolescents in the experimental group and $87.55 \%$ adolescents in the control group were in the age group of 13-14 years. $60 \%$ of the adolescents in experimental group were males in gender, whereas in the control group 50\% were males and 50\% were females. According to religion, $50 \%$ of the adolescents in experimental group and $85 \%$ of the adolescents in the control group belongs to Hindu religion. Majority of the adolescents in both the experimental $(95 \%)$ and control $(82.5 \%)$ groups belong to non-vegetarian by dietary pattern. $32.5 \%$ of the adolescents in the experimental group and $30 \%$ of adolescents in the control group belong to the monthly family income (in Indian rupees) between 16,020 and $32,049.40 \%$ of the adolescents in the experimental group and $22.5 \%$ adolescents in the control group had family history of high blood sugar, $20 \%$ of the adolescents in the experimental group and $25 \%$ in control group had family history of high blood pressure and $37.5 \%$ in experimental group and $22.5 \%$ in the control group had no family history of any diseases. $27.5 \%$ of the adolescents in the experimental group and $7.5 \%$ in the control group had family history of (parental) smoking. BMI was within normal range, waist and hip circumferences were within normal range and regarding blood pressure none of the children had hypertension.

\subsection{Section-II:}

3.2.1.: Distribution of adolescents according to pre-test score on knowledge regarding prevention of selected modifiable coronary artery disease risk factors in experimental and control groups.

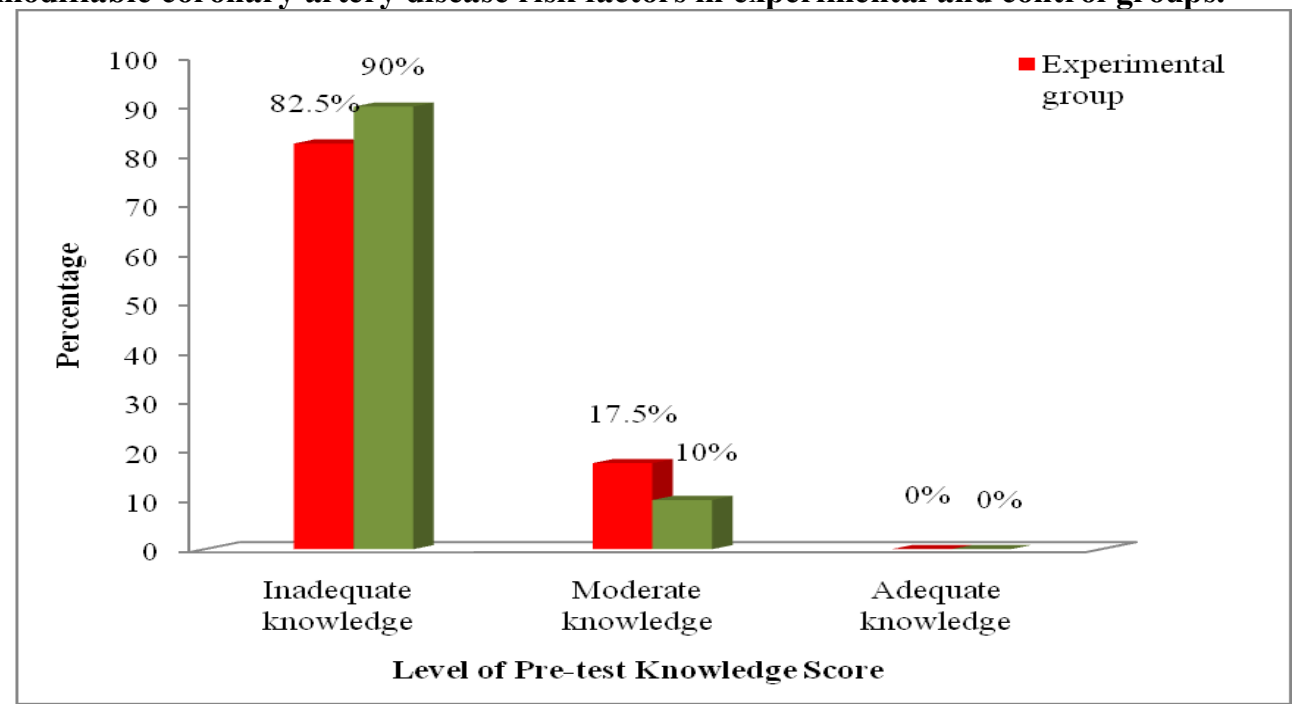

Fig-1: Level of pre-test knowledge scores in experimental and control groups.

The above figure depicts that majority $90 \%$ in control group and $82.5 \%$ in experimental group had inadequate knowledge. 
3.2.2: Distribution of adolescents according to pre-test scores on attitude regarding prevention of selected modifiable coronary artery disease risk factors in experimental and control groups.

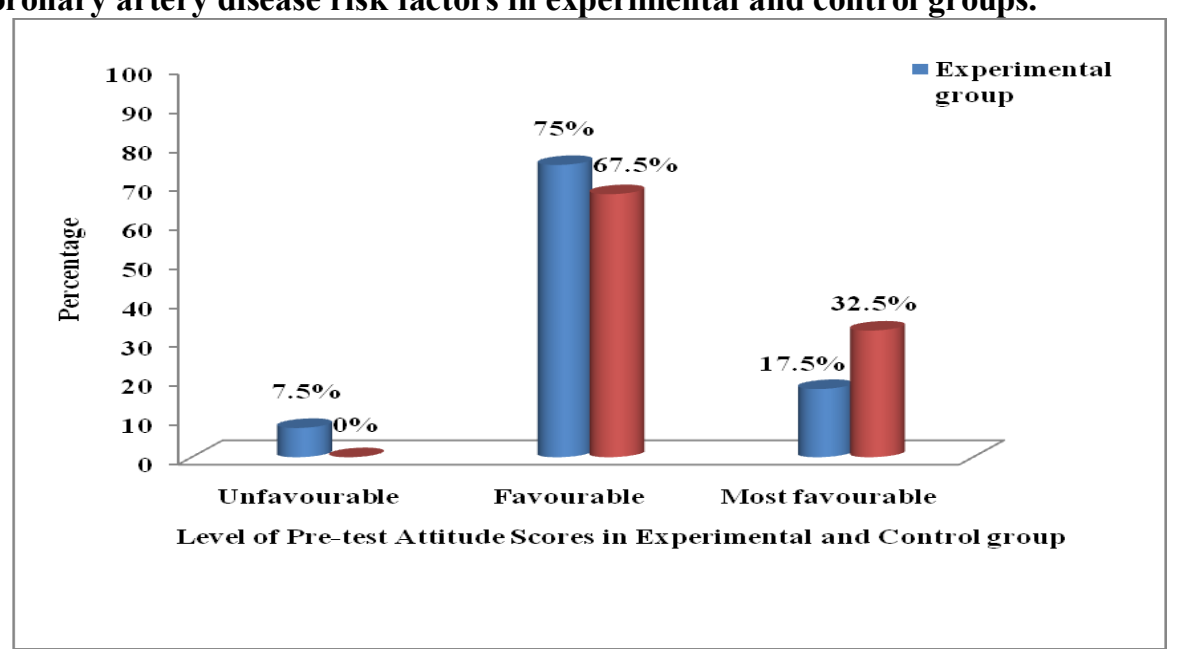

Fig-2: Level of pre-test attitude scores in experimental and control groups.

The above figure depicts that majority $75 \%$ in experimental group and $67.5 \%$ in control group had a favourable attitude.

3.2.3: Distribution of adolescents according to pre-test scores on health behavior regarding prevention of selected modifiable coronary artery disease risk factors in experimental and control groups.

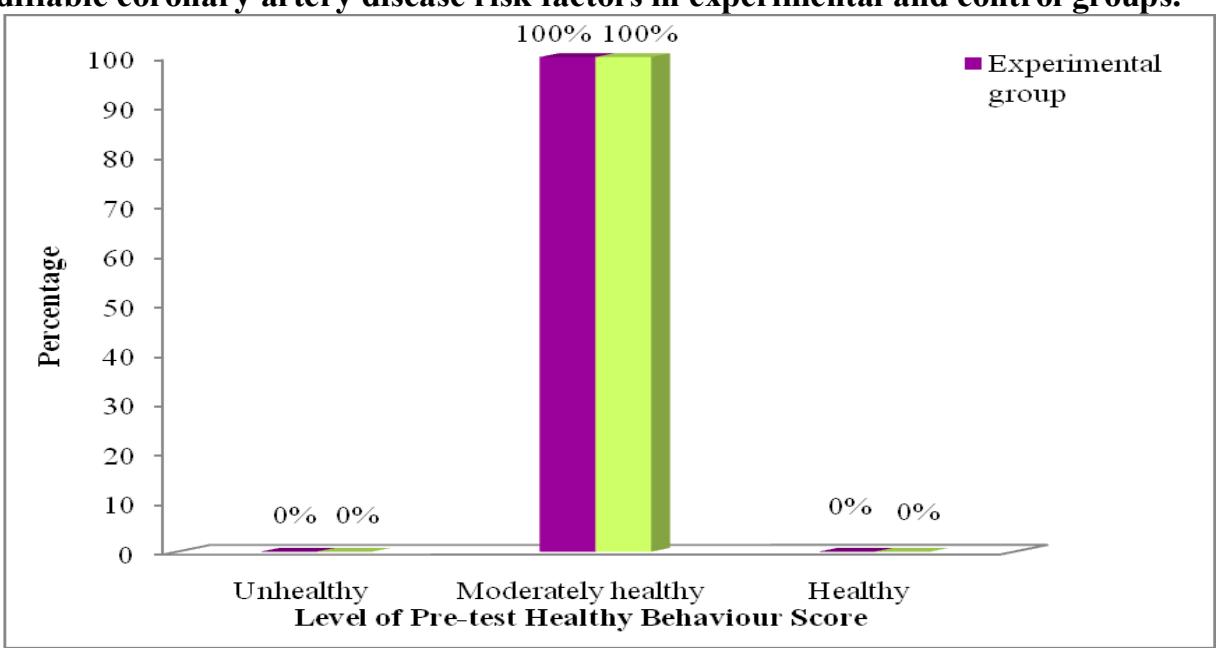

Fig-3: Level of pre-test health behaviour scores in experimental and control groups.

The above figure depicts, in both the experimental and control group none of them had either a healthy or unhealthy behavior.

3.3. Section-C: Evaluation of structured interventional strategy in terms of gain in knowledge, attitude and health behavior scores.

Table-1: Mean, SD, mean difference and ' $t$ ' value pre and $3^{\text {rd }}$ post-test scores of knowledge, attitude and health behavior in experimental group regarding prevention of selected modifiable coronary artery disease risk factors among adolescents.

\begin{tabular}{|c|c|c|c|c|c|c|c|}
\hline \multirow{2}{*}{ Variables } & \multicolumn{2}{|c|}{ Pre-test } & \multicolumn{2}{|c|}{$\mathbf{3}^{\text {rd }}$ Post-test } & \multirow{2}{*}{ Mean difference } & \multirow{2}{*}{ 't' value } & \multirow{2}{*}{ p-value } \\
\cline { 2 - 5 } & Mean & SD & Mean & SD & & 24.86 & $0.001^{* * *}$ \\
\hline Knowledge & 9.53 & 2.78 & 20.88 & 0.91 & 11.35 & 14.97 & $0.001 * * *$ \\
\hline Attitude & 68.55 & 9.09 & 90.9 & 2.69 & 22.35 & 27.01 & $0.001 * * * *$ \\
\hline Health behavior & 63.65 & 5.09 & 90.28 & 3.13 & 26.63 & 27.01 \\
\hline
\end{tabular}

(df $=39, * * *-\mathbf{p}<0.001$ highly significant) 
The above table shows that there was a significant difference in the $3^{\text {rd }}$ post-test scores on knowledge, attitude and health behavior and the calculated ' $\mathrm{t}$ ' value (paired) were highly significant at $\mathrm{P}<0.001$ level.

Table-2: Mean, SD, mean difference and ' $t$ ' value pre and $3^{\text {rd }}$ post-test scores of knowledge, attitude and health behavior in control group regarding prevention of selected modifiable coronary artery disease risk factors among adolescents.

\begin{tabular}{|c|c|c|c|c|c|c|c|}
\hline \multirow{2}{*}{ Variables } & \multicolumn{2}{|c|}{ Pre-test } & \multicolumn{2}{|c|}{$\mathbf{3}^{\text {rd }}$ Post-test } & \multirow{2}{*}{ Mean difference } & \multirow{2}{*}{ 't' value } & \multirow{2}{*}{ p-value } \\
\cline { 2 - 5 } & Mean & SD & Mean & SD & & 0.03 \\
\hline Knowledge & 9.03 & 2.61 & 8.03 & 1.69 & 1 & 2.19 & 0.732 \\
\hline Attitude & 72.28 & 6.72 & 71.1 & 6.89 & 1.18 & 0.468 \\
\hline Health behavior & 67.98 & 3.25 & 65.83 & 2.35 & 2.15 & 3.004 & 0.005 \\
\hline
\end{tabular}

$(\mathbf{d f}=\mathbf{3 9}, * * *-\mathbf{p}<\mathbf{0 . 0 0 1})$

The above table shows that there was no significant difference in the $3^{\text {rd }}$ post-test when comparing with the pretest and the calculated ' $t$ ' value had no significant results.

Table-3: Mean, SD, mean difference and ' $t$ ' value pre and $3^{\text {rd }}$ post-test scores of knowledge, attitude and health behavior in experimental and control group group regarding prevention of selected modifiable coronary artery disease risk factors among adolescents.

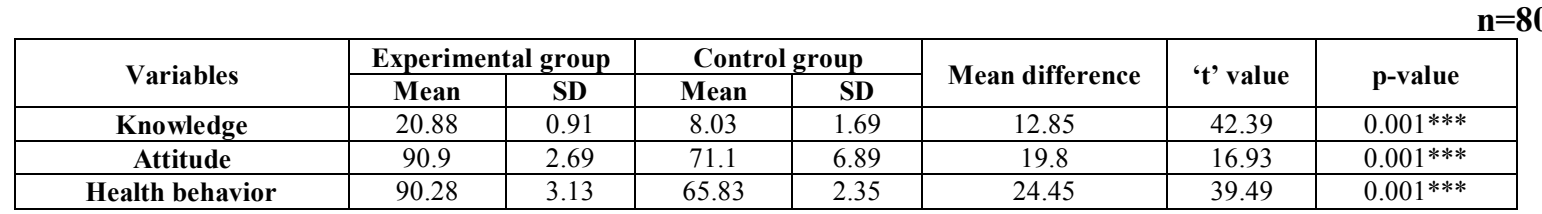

(df $=39, * * *-\mathbf{p}<\mathbf{0 . 0 0 1}$ highly significant)

The above table shows that there was a significant difference between the mean $3^{\text {rd }}$ post-test scores on knowledge, attitude and health behavior of the adolescents in experimental and control groups. The calculated ' $\mathrm{t}$ ' (unpaired) value was highly significant at $\mathrm{P}<0.001$ level.

In the $3^{\text {rd }}$ post-test mean scores of the experimental group on knowledge, attitude and health behavior were higher than the $3^{\text {rd }}$ post-test mean scores of control group.

The obtained ' $r$ ' values were not significant at $\mathrm{P}<0.001$ level neither in the pre-test nor in the post-test for both the experimental and control group. There was no significant correlation between knowledge - attitude, knowledge - health behavior, attitude and health behavior. Hence the research hypothesis $\mathrm{H}_{3}$ is rejected.

The calculated chi-square value did not show any association between the post-test score on knowledge with their selected demographic variables among adolescents except for dietary patterns $\left(\chi^{2}=17.09\right)$ significant at $\mathrm{P} \leq 0.001$ level in the control group. Hence the research hypothesis $\mathrm{H}_{4}$ was accepted only for this variable and rejected for all the other variables.

\section{Discussion}

Among 80 adolescents $52.5 \%$ of the adolescents in the experimental group and $87.55 \%$ adolescents in the control group were in the age group of $13-14$ years, $60 \%$ of the adolescents in the experimental group were males in gender, whereas in the control group 50\% were males and 50\% were females. According to religion, $50 \%$ of the adolescents in the experimental group and $85 \%$ of the adolescents in the control group belongs to Hindu religion. Majority of the adolescents in both the experimental (95\%) and control (82.5\%) groups belong to non-vegetarian by dietary pattern $32.5 \%$ of the adolescents in the experimental group and $30 \%$ of the adolescents in the control group belong to the monthly family income (in Indian rupees) between Rs.16,020 and 32,049 . In the experimental group $40 \%$ of the adolescents and $22.5 \%$ adolescents in the control group had family history of high blood sugar, $20 \%$ of the adolescents in the experimental group and $25 \%$ in control group had family history of high blood pressure and $37.5 \%$ in experimental group and $22.5 \%$ in the control group had no family history of any diseases. $27.5 \%$ of the adolescents in the experimental group and $7.5 \%$ in the control group had family history of paternal smoking. BMI, hip - waist circumference were within normal limits and none of the adolescents had hypertension. The present study findings is supported by the study of Rani Shetty, (2010) at Malad, North India. Among 600 adolescents studying $7^{\text {th }} 8^{\text {th }}$ and $9^{\text {th }}$ standards. 5\% had family history of heart disease, $7 \%$ had family history of diabetes mellitus, and $16 \%$ had family history of hypertension and $84 \%$ were non-vegetarians. ${ }^{(13)}$

With regard to knowledge, $82.5 \%$ of the adolescents in the experimental group and $90 \%$ of the adolescents in the control group had inadequate knowledge in the pre-test and $17.5 \%$ of the adolescents in the experimental group and $10 \%$ in the control group had moderate knowledge and none of them had adequate 
knowledge. With regard to attitude pre-test scores, $17.5 \%$ of adolescents had unfavourable attitude in the experimental group, $75 \%$ of adolescents in experimental group and $67.5 \%$ in the control had favourable attitude, none had the most favourable attitude. According to the pretest health behavior scores, none of the adolescents had neither unhealthy nor healthy behavior. The adolescents $(100 \%)$ in both the control and experimental groups had moderate healthy behavior.

The present study findings is supported by a cross -sectional study conducted by Nasir Aliya, (2011) to assess the knowledge and practice among 24 students (10 boys and 14 girls) selected from a school. The results revealed that majority of school children (66.7 and 62.5\%) reported that CVD's were the leading cause of death among the adults in Pakistan, while 25\% were not aware. Only 29.2\% agreed that CVD's were preventable. A significant majority of the participants $(62.5 \%)$ do not perform any physical exercise. Mean time spent on doing home work was 19-46 hours/ week and doing sedentary recreational activity was 15.80 hours/ week for both genders. Girls were remarkedly more overweight/ obese as compared to boys. ${ }^{(14)}$

In the experimental group: The overall paired ' $t$ ' test for pre and $3^{\text {rd }}$ post-test scores of knowledge was 24.86 at $\mathrm{P}<0.001$ levels which was highly significant. For attitude it was 14.97 at $\mathrm{P}<0.001$ level which was highly significant. For health behavior it was 27.01 at $\mathrm{P}<0.001$ level which was highly significant. Whereas in the control group: The overall paired ' $t$ ' test for pre and $3^{\text {rd }}$ post-test scores of knowledge was 2.19 , for a ' $p$ ' value of 0.03 . For attitude it was 0.732 for a ' $p$ ' value of 0.468 , for health behavior it was 3.004 for a 'p' value of 0.005 at $\mathrm{P}<0.001$ level. None of the values were significant.

Hypothesis testing: The overall unpaired ' $t$ ' test for $3^{\text {rd }}$ test scores between experimental and control group regarding prevention of selected modifiable CAD risk factors among adolescents. In the experimental group, the unpaired ' $\mathrm{t}$ ' value for knowledge was 42.39 at $\mathrm{P}<0.001$ level, highly significant, for attitude it was 16.93 at $\mathrm{P}<0.001$ level, highly significant, and for health behavior it was 39.49 at $\mathrm{P}<0.001$ level which was highly significant. There is a significant difference between the scores of experimental and control groups on knowledge, attitude and health behavior regarding prevention of selected modifiable CAD risk factors among adolescents. Hence, the research hypothesis $\mathrm{H}_{2}$ accepted.

Correlation between the knowledge, attitude and health behaviour regarding prevention of selected modifiable coronary artery disease risk factors. The post-test ' $r$ ' value for knowledge was $(-0.078)$, for attitude $(0.123)$ and health behavior $(-0.37)$. The obtained ' $r$ ' values were not significant at $\mathrm{P}<0.001$ level for any of the variables like knowledge, attitude and health behavior neither for experimental group nor for control group of adolescents. Hence the research hypothesis $\mathrm{H}_{3}$ was rejected.

The calculated chi-square value did not show any association between the post-test score on knowledge with their selected modifiable CAD risk factors among adolescents in the control group except for dietary patterns $\left(\chi^{2}-17.09\right)$ significant at $P \leq 0.001$ level. Hence the research hypothesis $\mathrm{H}_{4}$ was accepted only for this variable and rejected for all the other variables.

There was no significant association between the attitude, or health behavior and their selected demographic variables in both experimental and control groups. Hence, the research hypothesis $\mathrm{H}_{4}$ was rejected.

\section{Recommendations}

- A similar study can be done for a large samples to generalize the findings for a large population.

- A longitudinal study can be done to determine the effects of a planned teaching / video assisted teaching programme.

- A similar study can be done in different settings.

- A similar study can be done using bio-chemical tests such as lipid profile tests to estimate the serum cholesterol levels.

\section{Conclusion}

For prevention and control of CVD, unhealthy behaviours of people including tobacco use, unhealthy diet, physical inactivity and the harmful use of alcohol, need to be modified. These behaviours are shaped by economic growth, globalization, urbanization, social determinants and corporate influences, health literacy, individual efforts, and health education by health professionals can play an important role in modifying these behaviours. In the present study the video-assisted teaching intervention was very effective in improving the knowledge, attitude and developing a healthy behavior regarding prevention of selected modifiable CAD risk factors among adolescents.

\section{Acknowledgement}

I thank all the higher officials, principals and the students of the schools that have extended their cooperation in completing this study. 


\section{References}

[1]. WHO. Causes of death: 2008. Available from Url:http://www.who.int/ healthinfo./global_burden_ disease/CAD_2008_ sources_methods_pdf.

[2]. WHO. Causes of death: 2008. Available from Url:http://www.who.int/ healthinfo./global_burden_ disease/CAD_2008 sources_methods_pdf.

[3]. WHO. Global status report on non-communicable diseases. 2010. Available from Url:http://www.who.int/ globalstatusreportonNCD.

[4]. WHO. Global health risks: mortality and burden of diseases. 2010. Fact sheets [serial online]. Available from Url:http://www.who.int/ mortality.

[5]. Yusuf et.al, The inter heart case-control study, Lancet, 364, 2004, 937-52.

[6]. Mammi et.al, (1991). A 20 yrs hospital based study, Indian heart J. 199: 42(2): 93-6.

[7]. WHO. CVD: Prevention and control, 2006. Available from Url: http://www.who.int/mediacentre/factsheets/fs317/en/index.html.

[8]. $\quad$ Lewis Heit Kemper, Medical-surgical nursing, $7^{\text {th }}$ ed, NY:Mosby Elsevier, 2007.

[9]. CVD Atlas-WHO, (Geneva, 2012) Chapter 03-24, 25

[10]. CVD Atlas-WHO, (Geneva, 2012) Chapter 04-pdf-adobereader

[11]. Marilyn J. Hocken Berry, David Wilson, Health promotion of adolescent and family, Mosby Elsevier, $8^{\text {th }}$ ed, Wong's Essentials of Pediatric Nursing, (St. Louis, Missouri - 2009) 514-530.

[12]. Kleigmen, Stanton, St.Geme, Nelson Text book of Pediatrics, Elsevier Saunders: Philadelphia, 2011, 13-39.

[13]. Rani Shetty, CAD risk factors among adolescents, The Nursing Journal of India, July 2010, Vol. 7.

[14]. Alia Nasir, Assessment of CVD risk factors among school children in a private school, Karachi, Pakistan - A pilot study Url:http://www.handle.net/10755/201997. 